Marquette University

e-Publications@Marquette

Biomedical Engineering Faculty Research and

Publications

Biomedical Engineering, Department of

8-1-2004

\title{
Cardioprotection by Glucose-Insulin-Potassium: Dependence on KATP Channel Opening and Blood Glucose Concentration Before Ischemia
}

John F. LaDisa

Marquette University, john.ladisa@marquette.edu

John G. Krolikowski

Medical College of Wisconsin

Paul S. Pagel

Marquette University

David C. Warltier

Marquette University

Judy R. Kersten

Medical College of Wisconsin

Follow this and additional works at: https://epublications.marquette.edu/bioengin_fac

Part of the Biomedical Engineering and Bioengineering Commons

\section{Recommended Citation}

LaDisa, John F.; Krolikowski, John G.; Pagel, Paul S.; Warltier, David C.; and Kersten, Judy R., "Cardioprotection by Glucose-Insulin-Potassium: Dependence on KATP Channel Opening and Blood Glucose Concentration Before Ischemia" (2004). Biomedical Engineering Faculty Research and Publications. 216.

https://epublications.marquette.edu/bioengin_fac/216 
Marquette University

e-Publications@Marquette

\section{Biomedical Engineering Faculty Research and Publications/College of} Engineering

This paper is NOT THE PUBLISHED VERSION; but the author's final, peer-reviewed manuscript. The published version may be accessed by following the link in the citation below.

American Journal of Physiology : Heart and Circulatory Physiology, Vol. 287, No. 2 (August 2004): H601H607. DOI. This article is (C) American Physiological Society and permission has been granted for this version to appear in e-Publications@Marquette. American Physiological Society does not grant permission for this article to be further copied/distributed or hosted elsewhere without the express permission from American Physiological Society.

\section{Cardioprotection By Glucose-Insulin- Potassium: Dependence on K $\mathrm{K}_{\text {ATP }}$ Channel Opening and Blood Glucose Concentration Before Ischemia}

John F. LaDisa Jr.

Departments of Anesthesiology, Department of Biomedical Engineering, Marquette University, Milwaukee, Wisconsin

John G. Krolikowski

Departments of Anesthesiology, Department of Biomedical Engineering, Marquette University, Milwaukee, Wisconsin

Paul S. Pagel

Departments of Anesthesiology, Department of Biomedical Engineering, Marquette University, Milwaukee, Wisconsin 


\section{David C. Warltier}

Departments of Anesthesiology, Division of Cardiovascular Diseases, Department of Medicine, and Pharmacology and Toxicology, the Medical College of Wisconsin and the Clement J. Zablocki Veterans Affairs Medical Center Milwaukee Department of Biomedical Engineering, Marquette University, Milwaukee, Wisconsin Judy R. Kersten

Departments of Anesthesiology, Pharmacology and Toxicology, the Medical College of Wisconsin and the Clement J. Zablocki Veterans Affairs Medical Center, Milwaukee

Department of Biomedical Engineering, Marquette University, Milwaukee, Wisconsin

\section{Abstract}

We tested the hypothesis that glucose-insulin-potassium (GIK)-induced protection against myocardial infarction depends on ATP-dependent $\mathrm{K}^{+}\left(\mathrm{K}_{\text {ATP }}\right.$ ) channel activation and is abolished by hyperglycemia before the ischemia. Dogs were subjected to a 60-min coronary artery occlusion and 3-h reperfusion in the absence or presence of GIK (25\% dextrose; $50 \mathrm{IU}$ insulin/l; $80 \mathrm{mM} / \mathrm{I} \mathrm{KCl}$ infused at $1.5 \mathrm{ml} \cdot \mathrm{kg}^{-1} \cdot \mathrm{h}^{-1}$ ) beginning $75 \mathrm{~min}$ before coronary artery occlusion or $5 \mathrm{~min}$ before reperfusion. The role of $\mathrm{K}_{\mathrm{ATP}}$ channels was evaluated by pretreatment with glyburide $(0.1 \mathrm{mg} / \mathrm{kg})$. The efficacy of GIK was investigated with increases in blood glucose (BG) concentrations to 300 or $600 \mathrm{mg} / \mathrm{dl}$ or experimental diabetes (alloxan/streptozotocin). Infarct size (IS) was $29 \pm 2 \%$ of the area at risk in control experiments. GIK decreased $(P<0.05)$ IS when administered beginning 5 min before reperfusion. This protective action was independent of BG (13 \pm 2 and $12 \pm 2 \%$ of area at risk; $B G=80$ or 600 $\mathrm{mg} / \mathrm{dl}$, respectively) but was abolished in dogs receiving glyburide (30 $\pm 4 \%$ ), hyperglycemia before ischemia (27 $\pm 4 \%$ ), or diabetes ( $25 \pm 3 \%$ ). IS was unchanged by GIK when administered before ischemia independent of BG $(31 \pm 3,27 \pm 2$, and $35 \pm 3 \%$; $B G=80,300$, and $600 \mathrm{mg} / \mathrm{dl}$, respectively). The insulin component of GIK promotes cardioprotection by $\mathrm{K}_{\text {ATP }}$ channel activation. However, glucose decreases $\mathrm{K}_{\text {ATP }}$ channel activity, and this effect predominates when hyperglycemia is present before ischemia.

in the early 1960s, glucose-insulin-potassium (GIK) was first proposed as a treatment to reduce myocardial ischemic injury (26). Several clinical trials conducted since that initial report have indicated that GIK may improve outcome in patients with acute myocardial infarction $(6,8)$, but the use of GIK in this setting has not been widely accepted. GIK may produce beneficial effects during ischemia and reperfusion by optimizing substrate utilization (5). Alternatively, recent data $(10,15,16,24)$ suggest that insulin may be the primary component of GIK responsible for cardioprotection by favorably modulating cell survival pathways independent of metabolic effects. Unlike other modalities that activate endogenous signal transduction pathways to produce myocardial protection before the onset of ischemia, insulin-induced protective effects have been shown to occur at reperfusion (16).

A large body of experimental evidence accumulated over the past decade implicates ATP-regulated $\mathrm{K}^{+}\left(\mathrm{K}_{\mathrm{ATP}}\right)$ channels as central mediators of protection against reversible and irreversible ischemic injury. Activation of these channels is impaired by acute and chronic hyperglycemia $(17,21)$. Hyperglycemia is a major contributor to and an independent predictor of short- and long-term cardiovascular mortality in patients with and without diabetes mellitus (DM) (13). However, control of blood glucose (BG) concentration and the potentially deleterious consequences of hyperglycemia during administration of GIK have received relatively little attention. A study by Heng et al. (14) conducted before the advent of thrombolytic drugs demonstrated that patients with acute myocardial infarction who were treated with GIK suffered greater mortality than those who did not receive GIK. Notably, BG concentrations were very poorly controlled in the study by Heng et al. (14) and increased to levels as high as $600 \mathrm{mg} / \mathrm{dl}$ during the first $2 \mathrm{~h}$ of treatment in patients receiving GIK. A more recent 
study (22) conducted in patients undergoing off-pump coronary artery surgery demonstrated that GIK failed to reduce myocardial injury compared with placebo. This trial was subsequently discontinued after interim analysis revealed a possible increase in complication rate that may have been related to recalcitrant hyperglycemia in the GIK group. In the present investigation, we tested the hypothesis that GIK decreases myocardial infarct size (IS) in a canine model of coronary artery occlusion (CAO) when administered at reperfusion and that this beneficial effect requires activation of $K_{\text {ATP }}$ channels. In addition, we tested the hypothesis that the presence of acute or chronic hyperglycemia before the onset of ischemia abolishes any beneficial effect afforded by GIK.

\section{MATERIALS AND METHODS}

All experimental procedures and protocols used in this investigation were reviewed and approved by the Institutional Animal Care and Use Committee of the Medical College of Wisconsin. All procedures and protocols conformed to the American Physiological Society "Guiding Principles in the Care and Use of Animals" and the National Institutes of Health Guide for the Care and Use of Laboratory Animals (NIH Publication No. 85-23, Revised 1996).

\section{Acute surgical preparation.} Implantation of instruments has been previously described (20). Briefly, barbiturate-anesthetized dogs (18-22 $\mathrm{kg}$ ) were ventilated using positive pressure after endotracheal intubation. Arterial blood gas tensions and acidbase status were maintained within physiological limits by adjustment of tidal volume and respiratory rate. A fluid-filled catheter was inserted into the right femoral vein and artery for drug administration and withdrawal of reference blood flow samples, respectively. After thoracotomy was performed in the left fifth intercostal space, a dual micromanometer-tipped catheter was inserted into the left ventricle (LV) and ascending aorta for measurement of LV and arterial pressures, respectively, and the maximum rate of increase of LV pressure $\left(+\mathrm{dP} / \mathrm{d} t_{\max }\right)$. A 1.5- to 2-cm segment of the proximal left anterior descending coronary artery distal to the first diagonal branch was isolated, and a silk ligature was placed around the vessel to produce CAO and reperfusion. A fluid-filled catheter was inserted into the left atrium for radioactive microsphere injection. Temperature was maintained with a heating blanket. Hemodynamic data were continuously monitored on a polygraph and simultaneously digitized by using a computer interfaced with an analog-to-digital converter.

\section{Experimental protocol.}

All dogs were subjected to a 60 -min $\mathrm{CAO}$ and $3 \mathrm{~h}$ of reperfusion (Fig. 1). Control dogs received an intravenous infusion of saline $\left(1.5 \mathrm{ml} \cdot \mathrm{kg}^{-1} \cdot \mathrm{h}^{-1}\right)$. The effects of GIK (25\% dextrose; $50 \mathrm{IU}$ insulin/l; $80 \mathrm{mM} / \mathrm{I} \mathrm{KCl}$ infused intravenously at $1.5 \mathrm{ml} \cdot \mathrm{kg}^{-1} \cdot \mathrm{h}^{-1}$ ) (6) on IS were evaluated in two groups of dogs receiving GIK beginning 75 min before CAO ( $\mathrm{GIK}_{\mathrm{Con}}$ ) or $5 \mathrm{~min}$ before reperfusion (GIK reperfusion). Whether acute hyperglycemia modulated the actions of GIK was evaluated in three additional groups of dogs during infusion of $25 \%$ dextrose to increase BG to 300 or $600 \mathrm{mg} / \mathrm{dl}$ beginning $75 \mathrm{~min}$ before CAO (GIK 300 or $\mathrm{GIK}_{600}$ ) or $5 \mathrm{~min}$ before reperfusion (GIK 600 reperfusion). The impact of $\mathrm{K}_{\text {ATP }}$ channel activity on the protective effects of GIK was investigated in another group of dogs pretreated with glyburide $(0.1 \mathrm{mg} / \mathrm{kg}$, intravenously; Glb+GIK reperfusion) (20). Finally, the hypothesis that the presence of acute or chronic hyperglycemia abolishes the cardioprotection produced by GIK administered at reperfusion was examined in two separate groups of dogs, who received $25 \%$ dextrose to increase BG to $600 \mathrm{mg} / \mathrm{dl}$ beginning $75 \mathrm{~min}$ before CAO (Hyp+GIK reperfusion) or were subjected to chemically induced DM (DM+GIK reperfusion) (intravenous administration of $40 \mathrm{mg} / \mathrm{kg}$ alloxan and $25 \mathrm{mg} / \mathrm{kg}$ streptozotocin) (21). 


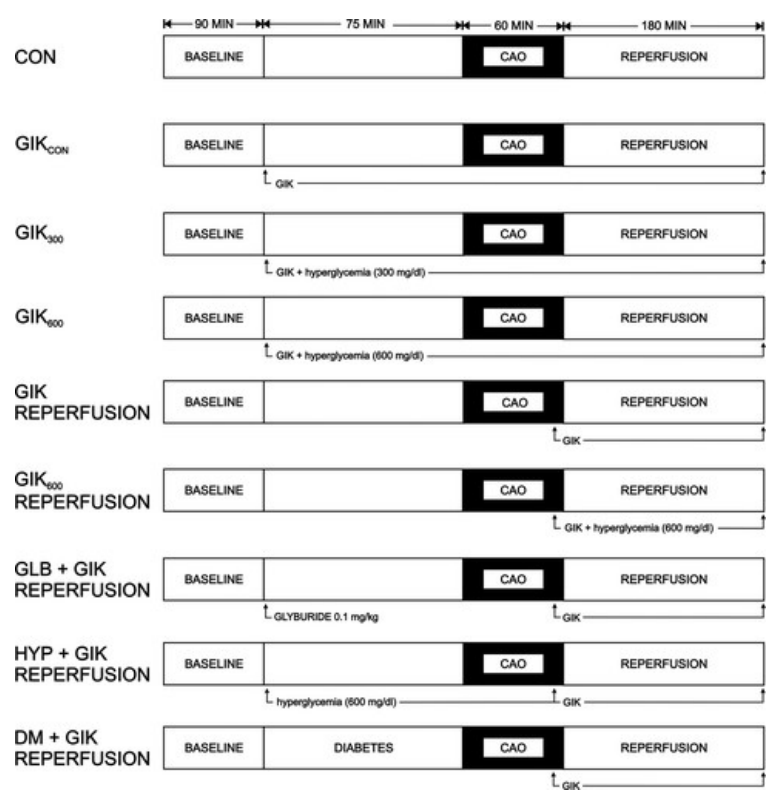

Fig. 1.Schematic illustration of the experimental protocol. GIK, glucose-insulin-potassium; GIK ${ }_{300}$, GIK given at $300 \mathrm{mg} / \mathrm{dl}$; GIK ${ }_{600}$, GIK given at 600 mg/dl; Glb, glyburide; Hyp, hyperglycemia; DM, diabetes mellitus; CAO, coronary artery occlusion; Con, saline control.

\section{Quantification of IS.}

IS was determined at the conclusion of the each experiment as previously described (29). Briefly, the LV area at risk (AAR) for infarction was separated from the normal area, and each region was incubated at $37^{\circ} \mathrm{C}$ for 20 to 30 min in 1\% 2,3,5-triphenyltetrazolium chloride in $0.1 \mathrm{M}$ phosphate buffer adjusted to $\mathrm{pH}$ 7.4. After overnight storage in $10 \%$ formaldehyde, infarcted and noninfarcted myocardium within the AAR was separated and weighed. IS was expressed as a percentage of the AAR.

\section{Quantification of regional myocardial blood flow.}

Regional myocardial blood flow was measured under baseline conditions, during CAO, and after $1 \mathrm{~h}$ of reperfusion using the radioactive microsphere technique as previously described (18). Myocardial blood flow was calculated as $Q_{r} \cdot C_{m} \cdot C_{r}^{-1}$, where $Q_{r}$ is the rate of withdrawal of the reference blood flow sample (in $\mathrm{ml} / \mathrm{min}$ ), $C_{\mathrm{m}}$ is the activity (in cpm/g) of the myocardial tissue sample, and $C_{\mathrm{r}}$ is the activity (in cpm) of the reference blood flow sample. Transmural myocardial blood flow was calculated as the average of subepicardial, midmyocardial, and subendocardial blood flows.

\section{Statistical analysis.}

Statistical analysis of data within and between groups was performed using ANOVA for repeated measures, followed by Student-Newman-Keuls test. A $P$ value $<0.05$ was considered statistically significant. All data are expressed as means $\pm \mathrm{SE}$.

\section{RESULTS}

Seventy-two dogs were instrumented to obtain 64 successful experiments. Four dogs were excluded from subsequent analysis because subendocardial collateral blood flow exceeded $0.15 \mathrm{ml} \cdot \mathrm{min}^{-1} \cdot \mathrm{g}^{-1}$ (one dog each in the $\mathrm{GIK}_{\mathrm{con}}, \mathrm{GIK}_{300}$, GIK reperfusion, and Hyp+GIK reperfusion groups). Four dogs were excluded due to intractable ventricular fibrillation during CAO (one each in the GIK $_{\text {con, }}$ GIK $\mathrm{K}_{600}$, GIK reperfusion, and Hyp+GIK reperfusion groups). One dog died after diabetes was chemically induced and was therefore not instrumented. 


\section{Systemic hemodynamics.}

No differences in systemic hemodynamics were observed between groups under baseline conditions or during CAO and reperfusion (Table 1). Transient increases in $\mathrm{LV}+\mathrm{dP} / \mathrm{d} t_{\max }$ were observed in dogs receiving GIK or dextrose alone before ischemia, but these increases were not sustained during CAO or after reperfusion. 
Table 1. Systemic hemodynamics

\begin{tabular}{|c|c|c|c|c|c|c|}
\hline & & & & Reperfusion, $\mathrm{h}$ & & \\
\hline & Baseline & Intervention & 30-min CAO & 1 & 2 & 3 \\
\hline \multicolumn{7}{|l|}{$\mathrm{HR}$, beats/min } \\
\hline Con & $132 \pm 5$ & $127 \pm 5$ & $124 \pm 5$ & $121 \pm 6$ & $128 \pm 5$ & $124 \pm 6$ \\
\hline $\mathrm{GIK}_{\text {con }}$ & $141 \pm 6$ & $135 \pm 4$ & $134 \pm 5$ & $127 \pm 6$ & $136 \pm 10$ & $133 \pm 6$ \\
\hline $\mathrm{GIK}_{300}$ & $128 \pm 6$ & $130 \pm 7$ & $119 \pm 6$ & $117 \pm 6$ & $117 \pm 6$ & $123 \pm 9$ \\
\hline $\mathrm{GIK}_{600}$ & $128 \pm 4$ & $129 \pm 4$ & $122 \pm 4$ & $118 \pm 4$ & $114 \pm 8^{*}$ & $113 \pm 7^{*}$ \\
\hline GIK reperfusion & $131 \pm 7$ & $124 \pm 6$ & $125 \pm 6$ & $116 \pm 5$ & $115 \pm 6$ & $112 \pm 8^{*}$ \\
\hline GIK $_{600}$ reperfusion & $128 \pm 3$ & $122 \pm 5$ & $117 \pm 5$ & $118 \pm 6$ & $119 \pm 7$ & $119 \pm 8$ \\
\hline Glb + GIK reperfusion & $129 \pm 7$ & $122 \pm 7$ & $127 \pm 5$ & $123 \pm 6$ & $120 \pm 7$ & $123 \pm 7$ \\
\hline Hyp + GIK reperfusion & $134 \pm 4$ & $132 \pm 4$ & $133 \pm 4$ & $128 \pm 7 *$ & $119 \pm 5 *$ & $122 \pm 6 *$ \\
\hline DM + GIK reperfusion & $138 \pm 5$ & $134 \pm 7$ & $129 \pm 9$ & $122 \pm 7 *$ & $121 \pm 7^{*}$ & $124 \pm 8^{*}$ \\
\hline \multicolumn{7}{|l|}{$\mathrm{MAP}, \mathrm{mmHg}$} \\
\hline Con & $100 \pm 4$ & $101 \pm 4$ & $92 \pm 5$ & $90 \pm 6$ & $91 \pm 5$ & $91 \pm 5$ \\
\hline $\mathrm{GIK}_{\text {con }}$ & $97 \pm 4$ & $102 \pm 7$ & $90 \pm 5$ & $99 \pm 4$ & $103 \pm 2$ & $97 \pm 3$ \\
\hline $\mathrm{GIK}_{300}$ & $93 \pm 4$ & $95 \pm 3$ & $75 \pm 4^{*}$ & $77 \pm 3^{*}$ & $81 \pm 6$ & $87 \pm 2$ \\
\hline $\mathrm{GIK}_{600}$ & $98 \pm 6$ & $103 \pm 5$ & $82 \pm 5^{*}$ & $84 \pm 5^{*}$ & $78 \pm 4^{*}$ & $74 \pm 4^{*}$ \\
\hline GIK reperfusion & $92 \pm 5$ & $96 \pm 4$ & $86 \pm 4$ & $97 \pm 6$ & $99 \pm 5$ & $95 \pm 6$ \\
\hline GIK $_{600}$ reperfusion & $101 \pm 4$ & $103 \pm 5$ & $88 \pm 6^{*}$ & $97 \pm 7$ & $101 \pm 6$ & $98 \pm 6$ \\
\hline Glb + GIK reperfusion & $92 \pm 4$ & $85 \pm 6$ & $80 \pm 6$ & $86 \pm 7$ & $86 \pm 6$ & $85 \pm 7$ \\
\hline Hyp + GIK reperfusion & $98 \pm 4$ & $104 \pm 3$ & $97 \pm 5$ & $97 \pm 6$ & $90 \pm 7$ & $88 \pm 6$ \\
\hline DM + GIK reperfusion & $101 \pm 5$ & $100 \pm 4$ & $87 \pm 4$ & $89 \pm 7$ & $91 \pm 6$ & $86 \pm 8$ \\
\hline \multicolumn{7}{|l|}{ LVSP, $\mathrm{mmHg}$} \\
\hline Con & $110 \pm 5$ & $110 \pm 4$ & $96 \pm 5$ & $91 \pm 6$ & $96 \pm 5$ & $95 \pm 5$ \\
\hline $\mathrm{GIK}_{\text {con }}$ & $108 \pm 5$ & $114 \pm 7$ & $98 \pm 5$ & $105 \pm 5$ & $109 \pm 4$ & $105 \pm 4$ \\
\hline $\mathrm{GIK}_{300}$ & $105 \pm 4$ & $105 \pm 3$ & $82 \pm 4^{*}$ & $83 \pm 3 *$ & $87 \pm 6^{*}$ & $93 \pm 3$ \\
\hline $\mathrm{GIK}_{600}$ & $110 \pm 6$ & $115 \pm 7$ & $87 \pm 5^{*}$ & $90 \pm 5^{*}$ & $84 \pm 4^{*}$ & $80 \pm 3^{*}$ \\
\hline GIK reperfusion & $103 \pm 5$ & $107 \pm 4$ & $93 \pm 3$ & $102 \pm 5$ & $105 \pm 6$ & $99 \pm 7$ \\
\hline GIK $_{600}$ reperfusion & $109 \pm 3$ & $113 \pm 4$ & $92 \pm 6^{*}$ & $102 \pm 8$ & $104 \pm 6$ & $100 \pm 6$ \\
\hline Glb + GIK reperfusion & $103 \pm 5$ & $97 \pm 6$ & $87 \pm 6 *$ & $93 \pm 6$ & $96 \pm 5$ & $95 \pm 6$ \\
\hline Hyp + GIK reperfusion & $108 \pm 4$ & $115 \pm 3$ & $101 \pm 6$ & $103 \pm 6$ & $97 \pm 8$ & $96 \pm 7$ \\
\hline
\end{tabular}




\begin{tabular}{|c|c|c|c|c|c|c|}
\hline DM + GIK reperfusion & $109 \pm 6$ & $109 \pm 3$ & $94 \pm 3$ & $98 \pm 6$ & $99 \pm 7$ & $94 \pm 8$ \\
\hline \multicolumn{7}{|l|}{ LVEDP, $\mathrm{mmHg}$} \\
\hline Con & $5 \pm 1$ & $5 \pm 1$ & $16 \pm 3 *$ & $21 \pm 4^{*}$ & $14 \pm 2$ & $14 \pm 2$ \\
\hline $\mathrm{GIK}_{\text {con }}$ & $5 \pm 1$ & $4 \pm 1$ & $14 \pm 4^{*}$ & $16 \pm 4^{*}$ & $16 \pm 5^{*}$ & $17 \pm 4^{*}$ \\
\hline $\mathrm{GIK}_{300}$ & $6 \pm 1$ & $6 \pm 1$ & $13 \pm 2 *$ & $14 \pm 2 *$ & $13 \pm 2 *$ & $14 \pm 2 *$ \\
\hline $\mathrm{GIK}_{600}$ & $7 \pm 1$ & $7 \pm 2$ & $20 \pm 4^{*}$ & $13 \pm 2$ & $11 \pm 2$ & $10 \pm 2$ \\
\hline GIK reperfusion & $8 \pm 1$ & $7 \pm 1$ & $12 \pm 2 *$ & $13 \pm 3^{*}$ & $13 \pm 3^{*}$ & $13 \pm 2 *$ \\
\hline GIK $_{600}$ reperfusion & $8 \pm 1$ & $7 \pm 2$ & $14 \pm 3 *$ & $17 \pm 2 *$ & $12 \pm 2$ & $11 \pm 2$ \\
\hline Glb + GIK reperfusion & $8 \pm 2$ & $6 \pm 2$ & $12 \pm 3$ & $14 \pm 3^{*}$ & $16 \pm 3^{*}$ & $14 \pm 4^{*}$ \\
\hline Hyp + GIK reperfusion & $8 \pm 1$ & $8 \pm 1$ & $17 \pm 1^{*}$ & $15 \pm 3^{*}$ & $11 \pm 1$ & $12 \pm 2$ \\
\hline DM + GIK reperfusion & $5 \pm 1$ & $5 \pm 1$ & $7 \pm 1$ & $8 \pm 1$ & $9 \pm 1^{*}$ & $10 \pm 1^{*}$ \\
\hline \multicolumn{7}{|l|}{$+\mathrm{dP} / \mathrm{d} t_{\max }, \mathrm{mmHg} / \mathrm{s}$} \\
\hline Con & $2,000 \pm 160$ & $2,100 \pm 150$ & $1,640 \pm 160 *$ & $1,400 \pm 80^{*}$ & $1,430 \pm 70^{*}$ & $1,370 \pm 80^{*}$ \\
\hline $\mathrm{GIK}_{\text {con }}$ & $1,940 \pm 110$ & $2,060 \pm 180$ & $1,350 \pm 90^{*}$ & $1,330 \pm 90^{*}$ & $1,440 \pm 160^{*}$ & $1,270 \pm 90 *$ \\
\hline $\mathrm{GIK}_{300}$ & $1,680 \pm 60$ & $2,110 \pm 170 *$ & $1,420 \pm 70$ & $1,350 \pm 100$ & $1,410 \pm 120$ & $1,540 \pm 150$ \\
\hline $\mathrm{GIK}_{600}$ & $1,850 \pm 180$ & $2,400 \pm 170 *$ & $1,570 \pm 130$ & $1,700 \pm 130$ & $1,530 \pm 130$ & $1,520 \pm 30$ \\
\hline GIK reperfusion & $1,730 \pm 120$ & $1,770 \pm 80$ & $1,600 \pm 130$ & $1,640 \pm 130$ & $1,550 \pm 130$ & $1,420 \pm 130^{*}$ \\
\hline GIK $_{600}$ reperfusion & $1,810 \pm 90$ & $1,860 \pm 160$ & $1,470 \pm 120 *$ & $1,720 \pm 140$ & $1,830 \pm 80$ & $1,690 \pm 120$ \\
\hline Glb + GIK reperfusion & $1,880 \pm 120$ & $1,880 \pm 220$ & $1,590 \pm 110$ & $1,640 \pm 80$ & $1,630 \pm 120$ & $1,560 \pm 80$ \\
\hline Hyp + GIK reperfusion & $1,890 \pm 90$ & $2,430 \pm 110^{*}$ & $2,000 \pm 90$ & $1,760 \pm 100$ & $1,670 \pm 110^{*}$ & $1,850 \pm 180$ \\
\hline DM + GIK reperfusion & $2,020 \pm 140$ & $2,040 \pm 80$ & $1,660 \pm 60^{*}$ & $1,620 \pm 140^{*}$ & $1,550 \pm 100^{*}$ & $1,440 \pm 80 *$ \\
\hline
\end{tabular}

Values are means \pm SE. CAO, coronary artery occlusion; Con, control; GIK, glucose-insulin-potassium; Glb, glyburide; Hyp, hyperglycemia; DM, diabetes mellitus; HR, heart rate; MAP, mean arterial pressure; LVSP and LVEDP, left ventricular systolic and end-diastolic pressures, respectively; $+\mathrm{dP} / \mathrm{d} t_{\text {max }}$ maximum rate of increase of LV pressure.

${ }^{*} P<0.05$, significantly different from baseline. 
BG and plasma insulin concentrations.

Chemically induced DM significantly $(P<0.05)$ increased BG (Table 2$)$ under baseline conditions ( $339 \pm 21 \mathrm{mg} / \mathrm{dl}$ ) compared with control $(88 \pm 66 \mathrm{mg} / \mathrm{dl})$ dogs and $B G$ remained elevated throughout the experiment. BG was unchanged in the GIK $\mathrm{con}_{\text {n }}$ and GIK reperfusion groups. BG was increased during administration of supplemental intravenous dextrose. Plasma insulin concentrations were decreased in diabetic $(2 \pm 1 \mu \mathrm{U} / \mathrm{ml})$ compared with control (11 $\pm 1 \mu \mathrm{U} / \mathrm{ml}$ ) dogs. GIK administered before ischemia (GIK $\mathrm{K}_{\mathrm{con}}$ ) or before reperfusion (GIK reperfusion) increased insulin concentration from $12 \pm 3$ and $10 \pm 1$ at baseline to $62 \pm 12$ and $79 \pm 8 \mu \mathrm{U} / \mathrm{ml}$, respectively, after $3 \mathrm{~h}$ of reperfusion. The magnitude of increase in the concentration of insulin during experimentation was dependent on the severity of hyperglycemia (e.g., insulin concentration increased to $542 \pm 129$ and $711 \pm 145$ $\mu \mathrm{U} / \mathrm{ml}$ after $3 \mathrm{~h}$ of reperfusion in the GIK 600 and Hyp+GIK groups, respectively). 
Table 2. Blood glucose, plasma insulin, and systemic potassium concentrations

\begin{tabular}{|c|c|c|c|c|c|}
\hline & & & & Reperfusion, $\mathrm{h}$ & \\
\hline & Baseline & Intervention & 30-min CAO & 1 & 3 \\
\hline \multicolumn{6}{|c|}{ Blood glucose concentration, mg/dl } \\
\hline Con & $88 \pm 6$ & $86 \pm 8$ & $93 \pm 10$ & $101 \pm 18$ & $98 \pm 13$ \\
\hline $\mathrm{GIK}_{\text {con }}$ & $74 \pm 5$ & $86 \pm 5$ & $89 \pm 9$ & $74 \pm 2$ & $78 \pm 7$ \\
\hline $\mathrm{GIK}_{300}$ & $86 \pm 4$ & $313 \pm 27 * \dagger$ & $332 \pm 16 *+$ & $332 \pm 20 * \dagger$ & $344 \pm 22 * \dagger$ \\
\hline $\mathrm{GIK}_{600}$ & $66 \pm 7$ & $580 \pm 11 * \dagger$ & $595 \pm 4 *+$ & $580 \pm 13 *+$ & $600 \pm 10 * \dagger$ \\
\hline GIK reperfusion & $81 \pm 5$ & $78 \pm 7$ & $71 \pm 8$ & $89 \pm 12$ & $78 \pm 7$ \\
\hline GIK $_{600}$ reperfusion & $84 \pm 5$ & $76 \pm 5$ & $78 \pm 3$ & $569 \pm 25 *+$ & $579 \pm 20 * \dagger$ \\
\hline Glb + GIK reperfusion & $86 \pm 4$ & $90 \pm 8$ & $99 \pm 7$ & $79 \pm 4$ & $96 \pm 10$ \\
\hline Hyp + GIK reperfusion & $94 \pm 4$ & $561 \pm 8 * \dagger$ & $585 \pm 7 *+$ & $564 \pm 36 * \dagger$ & $579 \pm 10 *+$ \\
\hline DM + GIK reperfusion & $339 \pm 21 \pm$ & $324 \pm 23 \pm$ & $294 \pm 23 \pm$ & $419 \pm 30 \pm$ & $411 \pm 42 \pm$ \\
\hline \multicolumn{6}{|l|}{ Insulin, $\mu \mathrm{U} / \mathrm{ml}$} \\
\hline Con & $11 \pm 1$ & $10 \pm 2$ & $9 \pm 2$ & $9 \pm 3$ & $6 \pm 1$ \\
\hline $\mathrm{GIK}_{\text {con }}$ & $12 \pm 3$ & $51 \pm 6 * \dagger$ & $67 \pm 9 * \dagger$ & $56 \pm 5 *+$ & $62 \pm 12 *+$ \\
\hline $\mathrm{GIK}_{300}$ & $21 \pm 11$ & $181 \pm 21 * \dagger$ & $195 \pm 37 * \dagger$ & $279 \pm 66 * \dagger$ & $356 \pm 78 * \dagger$ \\
\hline $\mathrm{GIK}_{600}$ & $11 \pm 2$ & $195 \pm 22 * \dagger$ & $357 \pm 90 *+$ & $417 \pm 81 * \dagger$ & $542 \pm 129 *+$ \\
\hline GIK reperfusion & $10 \pm 1$ & $9 \pm 1$ & $15 \pm 7$ & $89 \pm 15 * \dagger$ & $79 \pm 8 *+$ \\
\hline GIK $_{600}$ reperfusion & $17 \pm 4$ & $10 \pm 2$ & $11 \pm 1$ & $295 \pm 42 * \dagger$ & $258 \pm 92 * \dagger$ \\
\hline GIb + GIK reperfusion & $13 \pm 3$ & $58 \pm 12$ & $70 \pm 18 \pm$ & $209 \pm 37 \overline{* \dagger}$ & $248 \pm 55^{* \dagger}$ \\
\hline Hyp + GIK reperfusion & $12 \pm 3$ & $95 \pm 20 \pm$ & $156 \pm 30+$ & $379 \pm 71 *+$ & $711 \pm 145 *+$ \\
\hline DM + GIK reperfusion & $2 \pm 1 \pm$ & $1 \pm 0 \pm$ & $2 \pm 0 \pm$ & $63 \pm 14 *+$ & $64 \pm 6 *+$ \\
\hline
\end{tabular}

Values are means $\pm \mathrm{SE}$.

${ }^{*} P<0.05$, significantly different from baseline;

${ }^{\dagger} P<0.05$, significantly different from the respective value in control experiments. 


\section{Myocardial IS.}

There were no differences in LV AAR (data not shown) or coronary collateral blood flow (Table 3 ) between groups. IS was $29 \pm 2 \%(n=7)$ of AAR in control experiments (Fig. 2). GIK significantly decreased IS when administered at reperfusion, and this action was independent of BG [13 $\pm 2(n=7)$ and $12 \pm 2 \%(n=7)$ of AAR; BG $=80$ or $600 \mathrm{mg} / \mathrm{dl}$, respectively]. The protective effects of GIK were abolished in dogs pretreated with glyburide ( $30 \pm 4 \% ; n=8$ ). The salutary action of GIK was also eliminated in diabetic dogs $(25 \pm 3 \% ; n=7)$ and in dogs when hyperglycemia was present before ischemia $(27 \pm 4 \% ; n=7)$. GIK also failed to decrease IS when administered before ischemia $(31 \pm 3 \% ; n=7 ; \mathrm{BG}=80 \mathrm{mg} / \mathrm{dl})$, and this effect was independent of BG $[27 \pm 2(n=$ 7) and $35 \pm 3 \%(n=7) ; \mathrm{BG}=300$ or $600 \mathrm{mg} / \mathrm{dl}$, respectively].

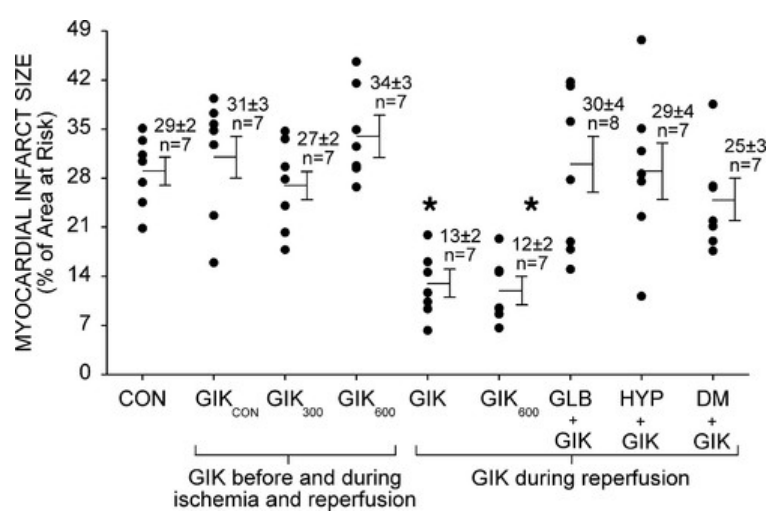

Fig. 2. Histograms illustrating myocardial infarct size expressed as a percentage of the left ventricular area at risk. Dogs were randomly subjected to saline (Con) or GIK administered before and during ischemia and reperfusion $\left(\mathrm{GIK}_{\mathrm{Con}}\right)$ or administered immediately before reperfusion. The effects of acute hyperglycemia to modulate the protection afforded by GIK were investigated in dogs receiving additional exogenous glucose to increase blood glucose to $\mathrm{GIK}_{300}, \mathrm{GIK} 600$, and Hyp + GIK. Other groups of dogs were subjected to experimental DM or received Glb. ${ }^{*} P<0.05$, significantly different from Con.

Table 3. Transmural myocardial perfusion in the ischemic region

\begin{tabular}{|l|c|c|c|}
\hline & Baseline & $30-$ min CAO & 1-h Reperfusion \\
\hline Con & $1.20 \pm 0.16$ & $0.07 \pm 0.01_{-}^{*}$ & $1.74 \pm 0.12_{-}^{*}$ \\
\hline GIK $_{\text {con }}$ & $1.29 \pm 0.22$ & $0.05 \pm 0.03_{-}^{*}$ & $1.57 \pm 0.12^{*}$ \\
\hline GIK $_{300}$ & $0.93 \pm 0.18$ & $0.11 \pm 0.10_{-}^{*}$ & $1.67 \pm 0.19_{-}^{*}$ \\
\hline GIK $_{600}$ & $1.09 \pm 0.15$ & $0.03 \pm 0.01_{-}^{*}$ & $1.73 \pm 0.25_{-}^{*}$ \\
\hline GIK reperfusion & $0.81 \pm 0.15$ & $0.09 \pm 0.02_{-}^{*}$ & $2.32 \pm 0.15_{-}^{*}$ \\
\hline GIK $_{600}$ reperfusion & $1.09 \pm 0.15$ & $0.11 \pm 0.02_{-}^{*}$ & $1.47 \pm 0.25^{*}$ \\
\hline GIb + GIK reperfusion & $0.77 \pm 0.17$ & $0.10 \pm 0.04_{-}^{*}$ & $1.58 \pm 0.34_{-}^{*}$ \\
\hline Hyp + GIK reperfusion & $0.90 \pm 0.16$ & $0.13 \pm 0.05_{-}^{*}$ & $1.99 \pm 0.35_{-}^{*}$ \\
\hline DM + GIK reperfusion & $0.73 \pm 0.10$ & $0.10 \pm 0.02_{-}^{*}$ & $2.37 \pm 0.25_{-}^{*}$ \\
\hline
\end{tabular}

Values are means $\pm \mathrm{SE}$. The perfusion rate was measured in $\mathrm{ml} \cdot \mathrm{min}-1 \cdot \mathrm{g}-1$. ${ }^{*} \mathrm{P}<0.05$, significantly different from baseline. 


\section{DISCUSSION}

\section{Cardioprotective signaling during GIK.}

The mechanisms by which GIK reduces ischemic injury are incompletely understood. It has become increasingly clear that insulin-activated signaling pathways are central features responsible for GIK-induced cardioprotection (24). Insulin alone decreased IS in isolated rat hearts, and this beneficial effect was abolished by inhibitors of protein tyrosine kinase, phosphatidylinositol-3-kinase, and p70s6 kinase (16). Insulin also promoted nitric oxide production (10) and stimulated phosphorylation of Akt as well as its downstream targets p70s6 and apoptotic regulator BCL2 antagonist of cell death (BAD) (16). These results supported previous findings demonstrating that insulin markedly decreased apoptosis by activating protein tyrosine kinase and phosphatidylinositol-3-kinase in a myocyte model of simulated ischemia and reoxygenation $(10,15)$. The beneficial effects of insulin contrast with the effects of hyperglycemia. Hyperglycemia has been shown to promote apoptotic myocyte death by increased production of angiotensin II with subsequent activation of p53 and p38 mitogen-activated protein kinases (9).

Recent evidence has also suggested a potential link between insulin signaling and $\mathrm{K}_{\text {ATP }}$ channel activation. $\mathrm{K}_{\text {ATP }}$ channels are critical mediators of ischemic preconditioning, a phenomenon responsible for powerful protection against myocardial infarction (11) and apoptosis (1). $\mathrm{K}_{\text {ATP }}$ channel activity is modulated by several intracellular kinases, including protein kinase $C$, protein tyrosine kinase, mitogen-activated protein kinases $(5,14)$, and phosphatidylinositol-3-kinase $(30)$. Insulin has also been shown to regulate $\mathrm{K}_{\mathrm{ATP}}$ channel activity $(27,29)$ by increasing the open-state probability of the channel and by decreasing channel sensitivity to ATP (28). Thus we hypothesized that insulin, administered as a component of GIK, produces cardioprotection by enhancing the activity of $\mathrm{K}_{\mathrm{ATP}}$ channels. We further hypothesized that the presence of hyperglycemia negatively influences this process $(17,19,21)$.

\section{GIK requires KaTP activation.}

The present results demonstrate for the first time that $\mathrm{K}_{\text {ATP }}$ channels mediate GIK-induced myocardial protection. The findings indicate that the nonselective $K_{\text {ATP }}$ channel antagonist glyburide abolishes salvage of ischemic and reperfused myocardium by GIK. Glyburide blocks mitochondrial and sarcolemmal KaTP channels that have both been identified to play roles during ischemic preconditioning, although the relative importance of each subcellular location of the channel remains somewhat controversial (12). The present results contrast with the findings of a previous report (4), suggesting that the selective mitochondrial $\mathrm{K}_{\mathrm{ATP}}$ channel antagonist 5hydroxydecanoate did not alter reductions in IS produced by a 5-min infusion of insulin 10 min before global ischemia in isolated rabbit hearts. Taken together, the present and previous data may implicate a role for the sarcolemmal $\mathrm{K}_{\text {ATP }}$ channel during cardioprotection with GIK. However, it is also possible that in the study of Baines et al. (4), 5-hydroxydecanoate administered during ischemia alone failed to block the beneficial effects of insulin because mitochondrial $\mathrm{K}_{\mathrm{ATP}}$ channel blockade was not sustained throughout ischemia and reperfusion. The models chosen to evaluate the role of $\mathrm{K}_{\mathrm{ATP}}$ channels during cardioprotection with GIK could also have influenced the results. We used intact, blood-perfused hearts with regional ischemia and reperfusion in anesthetized dogs, whereas Baines et al. (4) studied GIK in crystalloid perfused, globally ischemic, and reperfused isolated rabbit hearts.

\section{Hyperglycemia modulates GIK.}

The protective effects of GIK administered immediately before reperfusion occurred independent of BG during the reperfusion period as GIK was equally protective (IS $=13 \pm 2$ and $12 \pm 2 \%$ of $A A R$ ) in the presence of sevenfold differences in BG (78 \pm 7 and $579 \pm 20 \mathrm{mg} / \mathrm{dl}$, respectively). The present findings support previous observations in rats demonstrating that GIK or insulin alone administered immediately before reperfusion reduced IS, but glucose or potassium alone did not (10). The beneficial effects of insulin also persisted when pyruvate was substituted for glucose in this model, suggesting that substrate-specific metabolism may not be 
responsible for insulin-dependent cardioprotection (16). Interestingly, our results demonstrate that the presence of acute or chronic hyperglycemia before ischemia completely abolished the protective effects of GIK on reperfusion. The mechanism for this effect is not entirely clear but may involve adverse modulation of $\mathrm{K}_{\text {ATP }}$ channels by glucose. We (17) have shown that acute hyperglycemia or DM impairs activation of mitochondrial $K_{\text {ATP }}$ channels, and this action is dependent on both the severity of hyperglycemia and the dose of $\mathrm{K}_{\text {ATP }}$ channel agonist. Acute and chronic hyperglycemia blocks ischemic preconditioning $(17,19)$ and produces dose-related increases in myocardial IS (17). In contrast to our earlier findings, we did not observe hyperglycemia-induced increases in IS in the present study when exogenous insulin was also administered. Although IS was not reduced by insulin in the presence of preischemic moderate or severe hyperglycemia, insulin may have mitigated the deleterious effects of hyperglycemia that might otherwise have increased the extent of myocardial ischemic injury. The previous and present results support the contention that glucose, a known regulator of pancreatic $K_{\text {ATP }}$ channels (2), impairs activation of myocardial $K_{\text {ATP }}$ channels. Increasing glucose concentration within a physiological range from 3 to $6.5 \mathrm{mM}$ is sufficient to close $K_{\text {ATP }}$ channels within 13 min in pancreatic islet cells (23). This effect occurs concomitantly with increases in ATP concentration generated by oxidative substrate metabolism. Although the effects of glucose to regulate mitochondrial $\mathrm{K}_{\text {ATP }}$ channels have not been specifically investigated, it is likely that ATP generated from glucose metabolism also regulates channels in this organelle. Activation of $\mathrm{K}_{\mathrm{ATP}}$ channels during ischemia appears to be required to elicit insulin-dependent cardioprotection on reperfusion. The finding that hyperglycemia, when present only during reperfusion, does not attenuate GIK cardioprotection was somewhat surprising and suggests that opening of $\mathrm{K}_{\text {ATP }}$ channels during ischemia may serve as an initial trigger for an effector that is subsequently modulated by insulin in a glucose-independent fashion. Candidates for such an effector may include mitogenactivated protein kinases, other downstream kinases, or nitric oxide, all components of signaling pathways that have previously been shown to be modulated by insulin or $\mathrm{K}_{\mathrm{ATP}}$ channels $(10,12)$.

\section{Beneficial effects of GIK occur at reperfusion.}

Clinical and experimental evidence indicates that the protective effects of insulin (as a component of GIK) occur predominantly during reperfusion $(6,10,15,16)$. The current results support this contention and demonstrate that GIK does not alter IS when administered before and during ischemia but substantially decreases injury when given immediately before reperfusion. The efficacy of GIK may be modulated under these conditions because of glucose-impaired $\mathrm{K}_{\text {ATP }}$ channel activation or due to metabolic effects of insulin and glucose. For example, preischemic glycogen depletion improves metabolic and contractile recovery in preconditioned rat hearts, and this effect may be related to decreased proton production from a reduction in hydrolysis of glycogen-derived ATP (25). Conversely, it has been suggested that administration of insulin and glucose before ischemia may increase glycogen stores and paradoxically increase the extent of ischemic damage, thus precluding any beneficial effect of insulin at reperfusion (16). The hypothesis that glycogen depletion is beneficial before ischemia and reperfusion is not supported by other evidence in the literature, however. Resynthesis of glycogen on reperfusion was correlated with the return of contractile function in isolated rat hearts subjected to global ischemia and reperfusion (7). Nonetheless, it was not our intention to characterize the complex metabolic effects of GIK but to explore the interrelationships among insulin, glucose, and $\mathrm{K}_{\text {ATP }}$ channels as modulators of ischemia and reperfusion injury during GIK.

\section{Limitations and conclusions.}

The present results should be interpreted within the constraints of several potential limitations. The LV AAR for infarction and coronary collateral blood flow, important determinants of the extent of infarction, were similar between groups and do not account for the observed results. The dose of glyburide used in the present investigation did not affect IS in a previous study (18). The dose of GIK was based on the regimen demonstrated to be most efficacious in the Estudios Cardiologicos Latinoamerica GIK pilot clinical trial (6). Hyperglycemia 
produced by administration of dextrose before ischemia caused transient increases in LV $+\mathrm{dP} / \mathrm{d} t_{\max }$, an index of global myocardial contractility that is a major determinant of myocardial oxygen consumption. The increase in $\mathrm{LV}+\mathrm{dP} / \mathrm{d} t_{\text {max }}$, in hyperglycemic dogs before ischemia suggests that ischemic burden may have been greater in these experimental groups. However, there were no differences in $\mathrm{LV}+\mathrm{dP} / \mathrm{d} t_{\max }$ among groups, and it is unlikely that differences in the determinants of myocardial oxygen consumption among groups were solely responsible for the observed findings. Nevertheless, myocardial oxygen consumption was not directly measured in the current investigation.

In summary, the present results confirm that GIK reduces myocardial IS when administered immediately before reperfusion in a canine model of prolonged coronary occlusion and reperfusion. This beneficial effect is dependent on opening of $\mathrm{K}_{\mathrm{ATP}}$ channels during the ischemic period and is blocked by acute or chronic DM hyperglycemia before ischemia. The results reaffirm the temporal dependence of GIK administration and demonstrate for the first time that $\mathrm{K}_{\text {ATP }}$ channels mediate GIK-induced cardioprotection in vivo.

\section{GRANTS}

This work was supported by National Institutes of Health Grants HL-063705 (to J. R. Kersten), HL-054820, GM066730, and GM-008377 (all to D. C. Warltier).

\section{FOOTNOTES}

- The costs of publication of this article were defrayed in part by the payment of page charges. The article must therefore be hereby marked "advertisement" in accordance with 18 U.S.C. Section 1734 solely to indicate this fact.

The authors thank Mary Lorence-Hanke (Department of Anesthesiology, Medical College of Wisconsin) for assistance in preparation of the manuscript.

\section{AUTHOR NOTES}

- Address for reprint requests and other correspondence: J. R. Kersten, Medical College of Wisconsin, MEB-M4280, 8701 Watertown Plank Rd., Milwaukee, WI 53226 (E-mail: jkersten@mcw.edu).

\section{REFERENCES}

1 Akao M, Ohler A, O'Rourke B, and Marban E. Mitochondrial ATP-sensitive potassium channels inhibit apoptosis induced by oxidative stress in cardiac cells. Circ Res 88: 1267-1275, 2001.

2 Ashcroft FM, Harrison DE, and Ashcroft SJ. Glucose induces closure of single potassium channels in isolated rat pancreatic $\beta$-cells. Nature 312: 446-448, 1984.

3 Baines CP, Pass JM, and Ping P. Protein kinases and kinase-modulated effectors in the late phase of ischemic preconditioning. Basic Res Cardiol 96: 207-218, 2001.

4 Baines CP, Wang L, Cohen MV, and Downey JM. Myocardial protection by insulin is dependent on phospatidylinositol 3-kinase but not protein kinase $\mathrm{C}_{\text {or }} \mathrm{K}_{\text {AтP }}$ channels in the isolated rabbit heart. Basic Res Cardiol 94: 188-198, 1999.

5 Depre C, Vanoverschelde JL, and Taegtmeyer H. Glucose for the heart. Circulation 99: 578-588, 1999.

6 Diaz R, Paolasso EA, Piegas LS, Tajer CD, Moreno MG, Corvalan R, Isea JE, and Romero G. Metabolic modulation of acute myocardial infarction. The ECLA (Estudios Cardiologicos Latinoamerica) Collaborative Group. Circulation 98: 2227-2234, 1998.

7 Doenst T, Guthrie PH, Chemnitius JM, Zech R, and Taegtmeyer H. Fasting, lactate, and insulin improve ischemia tolerance in rat heart: a comparison with ischemic preconditioning. Am J Physiol Heart Circ Physiol 270: H1607-H1615, 1996. 
8 Fath-Ordoubadi F and Beatt KJ. Glucose-insulin-potassium therapy for treatment of acute myocardial infarction: an overview of randomized placebo-controlled trials. Circulation 96: 1152-1156, 1997.

9 Fiordaliso F, Leri A, Cesselli D, Limana F, Safai B, Nadal-Ginard B, Anversa P, and Kajstura J. Hyperglycemia activates p53 and p53-regulated genes leading to myocyte cell death. Diabetes 50: 2363-2375, 2001.

10 Gao F, Gao E, Yue TL, Ohlstein EH, Lopez BL, Christopher TA, and Ma XL. Nitric oxide mediates the antiapoptotic effect of insulin in myocardial ischemia-reperfusion: the roles of PI3-kinase, Akt, and endothelial nitric oxide synthase phosphorylation. Circulation 105: 1497-1502, 2002.

11 Gross GJ and Auchampach JA. Blockade of ATP-sensitive potassium channels prevents myocardial preconditioning in dogs. Circ Res 70: 223-233, 1992.

12 Gross GJ and Peart JN. K $\mathrm{ATT}_{\text {AP }}$ channels and myocardial preconditioning: an update. Am J Physiol Heart Circ Physiol 285: H921-H930, 2003.

13 Grundy SM, Benjamin IJ, Burke GL, Chait A, Eckel RH, Howard BV, Mitch W, Smith SC Jr, and Sowers JR. Diabetes and cardiovascular disease: a statement for healthcare professionals from the American Heart Association. Circulation 100: 1134-1146, 1999.

14 Heng MK, Norris RM, Singh BN, and Barratt-Boyes C. Effects of glucose and glucose-insulin-potassium on haemodynamics and enzyme release after acute myocardial infarction. Br Heart J 39: 748-757, 1977.

15 Jonassen AK, Brar BK, Mjos OD, Sack MN, Latchman DS, and Yellon DM. Insulin administered at reoxygenation exerts a cardioprotective effect in myocytes by a possible anti-apoptotic mechanism. J Mol Cell Cardiol 32: 757-764, 2000.

16 Jonassen AK, Sack MN, Mjos OD, and Yellon DM. Myocardial protection by insulin at reperfusion requires early administration and is mediated via Akt and p70s6 kinase cell-survival signaling. Circ Res 89: 1191-1198, 2001.

17 Kersten JR, Montgomery MW, Ghassemi T, Gross ER, Toller WG, Pagel PS, and Warltier DC. Diabetes and hyperglycemia impair activation of mitochondrial K KAT channels. Am J Physiol Heart Circ Physiol 280: H1744H1750, 2001.

18 Kersten JR, Schmeling TJ, Hettrick DA, Pagel PS, Gross GJ, and Warltier DC. Mechanism of myocardial protection by isoflurane. Role of adenosine triphosphate-regulated potassium (К $\mathrm{K}_{\text {AтP }}$ ) channels. Anesthesiology 85: 794807, 1996.

19 Kersten JR, Schmeling TJ, Orth KG, Pagel PS, and Warltier DC. Acute hyperglycemia abolishes ischemic preconditioning in vivo. Am J Physiol Heart Circ Physiol 275: H721-H725, 1998.

20 Kersten JR, Schmeling TJ, Pagel PS, Gross ER, and Warltier DC. Isoflurane mimics ischemic preconditioning via activation of $\mathrm{K}_{\text {AтP }}$ channels: reduction of myocardial infarct size with an acute memory phase. Anesthesiology 87: 361-370, 1997.

21 Kersten JR, Toller WG, Gross ER, Pagel PS, and Warltier DC. Diabetes abolishes ischemic preconditioning: role of glucose, insulin, and osmolality. Am J Physiol Heart Circ Physiol 278: H1218-H1224, 2000.

22 Lell WA, Nielsen VG, McGiffin DC, Schmidt FE Jr, Kirklin JK, and Stanley AW Jr. Glucose-insulin-potassium infusion for myocardial protection during off-pump coronary artery surgery. Ann Thorac Surg 73: 1246-1251, 2002.

23 Misler S, Gee WM, Gillis KD, Scharp DW, and Falke LC. Metabolite-regulated ATP-sensitive K+ channel in human pancreatic islet cells. Diabetes 38: 422-427, 1989.

24 Sack MN and Yellon DM. Insulin therapy as an adjunct to reperfusion after acute coronary ischemia: a proposed direct myocardial cell survival effect independent of metabolic modulation. J Am Coll Cardiol 41: 14041407, 2003.

25 Soares PR, de Albuquerque CP, Chacko VP, Gerstenblith G, and Weiss RG. Role of preischemic glycogen depletion in the improvement of postischemic metabolic and contractile recovery of ischemia-preconditioned rat hearts. Circulation 96: 975-983, 1997.

26 Sodi-Pallares D, Testelli MR, Fishleder BL, Bisteni A, Medrano GA, Friedland C, and De Micheli A. Effects of an intravenous infusion of a potassium-glucose-insulin solution on the electrocardiographic signs of myocardial infarction. A preliminary clinical report. Am J Cardiol 9: 166-181, 1962.

27 Spanswick D, Smith MA, Mirshamsi S, Routh VH, and Ashford ML. Insulin activates ATP-sensitive K+ channels in hypothalamic neurons of lean, but not obese rats. Nat Neurosci 3: 757-758, 2000.

28 Tricarico D, Mallamaci R, Barbieri M, and Conte Camerino D. Modulation of ATP-sensitive $\mathrm{K}^{+}$channel by insulin in rat skeletal muscle fibers. Biochem Biophys Res Commun 232: 536-539, 1997. 
29 Warltier DC, Zyvoloski MG, Gross GJ, Hardman HF, and Brooks HL. Determination of experimental myocardial infarct size. J Pharmacol Methods 6: 199-210, 1981.

30 Xie LH, Takano M, Kakei M, Okamura M, and Noma A. Wortmannin, an inhibitor of phosphatidylinositol kinases, blocks the MgATP-dependent recovery of Kir6.2/SUR2A channels. J Physiol 514: 655-665, 1999. 